\title{
Design of a Random Access Network for Compressed Sensing
}

\author{
Fatemeh Fazel \\ Northeastern University \\ Boston, MA, 02115 \\ Email: ffazel@ece.neu.edu
}

\author{
Maryam Fazel \\ University of Washington \\ Seattle, WA, 98195 \\ Email:mfazel@ee.washington.edu
}

\author{
Milica Stojanovic \\ Northeastern University \\ Boston, MA, 02115 \\ Email: millitsa@ece.neu.edu
}

\begin{abstract}
For networks that are deployed for long-term monitoring of environmental phenomena, it is of crucial importance to design an efficient data gathering scheme that prolongs the lifetime of the network. To this end, we exploit the sparse nature of the monitored field and consider a Random Access Compressed Sensing (RACS) scheme in which the sensors transmit at random to a fusion center which reconstructs the field. We provide an analytical framework for system design that captures packet collisions due to random access as well as packet errors due to communication noise. Through analysis and examples, we demonstrate that recovery of the field can be attained using only a fraction of the resources used by a conventional TDMA network, while employing a scheme which is simple to implement and requires no synchronization.
\end{abstract}

\section{INTRODUCTION}

Wireless sensor networks deployed for the purpose of environmental monitoring, surveillance, or data collection, have to be designed for a long life-time. Sensor nodes are typically distributed randomly or uniformly over a certain region and each sensor communicates its observations to a central node, referred to as the fusion center (FC). The FC then reconstructs the map of the field of interest. Once the network is deployed, there is little access to the sensors and hence re-charging batteries becomes difficult. Therefore, in battery-powered networks, network life-time is of utmost importance. To be able to operate over long intervals of time, sensor nodes need to conserve their energy. A large body of literature examines energy-aware design methodologies for managing the periodic sleep cycles of sensors, such as [1], [2], [3]. Moreover, in some networks, such as underwater acoustic networks, bandwidth is severely limited, hence efficient networking schemes are of particular importance [4], [5].

To achieve the desired energy and bandwidth efficiency, we capitalize on the fact that most natural phenomena are sparse (compressible) in an appropriate basis and exploit the principle of compressed sensing. The theory of compressed sensing establishes that under certain conditions, exact signal recovery is possible with a relatively small number of random measurements [6], [7]. Application of compressed sensing in wireless sensor networks was first introduced in [8], [9], [10], where the authors used phase-coherent transmission of randomlyweighted data from sensor nodes to the FC over a dedicated

Research funded in part by ONR grant N00014-09-1-0700, NSF grant 0831728, and NSF CAREER grant ECCS-0847077. multiple-access channel, to form distributed projections of data onto an appropriate basis at the FC. In this approach sensors need to be perfectly synchronized. In [11] authors consider a decentralized network (without FC), where active nodes exchange measurements locally. The authors formulate sparse recovery as a decentralized consensus optimization problem and show that their iterative algorithm converges to a globally optimal solution. Reference [12] proposes compressive cooperative mapping using mobile sensors based on a small set of observations. Authors in [13] consider spatial mapping by robots and propose an efficient way to reconstruct natural fields using random-walk-based sampling and compressed sensing.

Inspired by compressed sensing and employing random channel access, in [14] we proposed a simple and efficient networking scheme referred to as Random Access Compressed Sensing (RACS). The RACS scheme consists of distributed random sampling, followed by random channel access. The key idea behind RACS is that packet collisions (which are inevitable in random access) occur randomly and thus do not change the random nature of the observations provided to the FC. Since the FC only needs to receive some, and not all the sensor packets, it can simply disregard the collisions. By disregarding collisions, we eliminate the need for listening to the medium (as used in contention-based MAC protocols), which further reduces the energy consumption of the sensor nodes. The FC obtains an incomplete set of measurements (due to both random sensing and losses due to random access) from which it reconstructs the field using compressed sensing techniques. To provide a sufficient number of measurements to the FC, we compensate for the collision losses by initially selecting the number of participating sensors to be somewhat greater than the minimum number of packets required. The RACS scheme [14] assumes a frame-based (slotted) transmission that requires the sensor nodes to be synchronized. In certain network structures, such as large acoustic networks, maintaining synchronization is challenging due to variable propagation delays and clock drift. This fact motivates our present work, whose goal is to dispense with synchronization and scheduling requirements. Moreover, in the present work we address communication noise in the RACS network.

In this paper, we propose a RACS networking scheme that obviates the need for synchronized frame-based transmission. Knowing that the communication noise in an AWGN channel 
gives rise to packet errors, we let the FC discard the packets that are received in error, in addition to those that resulted in collision. To account for the packet losses that occur due to both collisions and noise, the network design employs the concept of sufficient sensing probability. With this probability, which defines the desired system performance, sufficiently many data packets - as required for field reconstruction based on compressed sensing - are to be received. We develop an analytical model for the data collection process and use this model to determine the transmission rate necessary to achieve the desired probability of sufficient sensing. The proposed continuous-time RACS has a simple implementation, eliminates the need for time-synchronization and scheduling, and achieves considerable savings in energy and bandwidth over conventional TDMA networks.

The rest of the paper is organized as follows. In Section II we introduce the RACS network model. In Section III we propose an analytical framework to model the arrival of useful packets to the FC. In Section IV we outline a design methodology to determine the network parameters. In Section $\mathrm{V}$ bandwidth and energy consumption of a RACS network are discussed. Section VI provides a comparison benchmark, while Section VII quantifies the savings in bandwidth and energy expenditure attained by employing RACS. Finally, we provide concluding remarks in Section VIII.

Notation: We denote by $\ell_{p}$ the $p$-norm of a vector $\mathbf{x}=\left[x_{1}, \ldots, x_{N}\right]^{T},\|\mathbf{x}\|_{\ell_{p}}=\left(\sum_{i=1}^{N}\left|x_{i}\right|^{p}\right)^{1 / p}$. If $\mathbf{V}$ is a $k \times l$ matrix, $\operatorname{vec}(\mathbf{V})$ denotes the $k l \times 1$ vector formed by stacking the columns of matrix $\mathbf{V}, \operatorname{vec}(\mathbf{V})=$ $\left[\begin{array}{lllllll}v_{11} & \ldots & v_{l 1} & \ldots & v_{1 k} & \ldots & v_{l k}\end{array}\right]^{T}$. Finally, $\mathbf{A} \otimes \mathbf{B}$ denotes the Kronecker product of matrices $\mathbf{A}$ and $\mathbf{B}$.

\section{RACS System Model}

Consider a grid network shown in Fig. 1, which consists of $N=I J$ sensors uniformly distributed on a two-dimensional plane, with $J$ and $I$ sensors in $x$ and $y$ directions, respectively. The network measures a physical phenomenon, $u(x, y, t)$, whose coherence time is $T_{c o h}$. At time $t$, the sensor node located at position $(i, j)$ in the network grid acquires a measurement $u_{i j}(t)=u\left(x_{j}, y_{i}, t\right)$. Since the process is slowly varying during $T_{c o h}$, we assume that $u_{i j}\left(t_{1}\right) \approx u_{i j}\left(t_{2}\right)$ for $\left|t_{1}-t_{2}\right| \leq T_{c o h}$. In what follows, we will focus on an observation window of size $T \leq T_{c o h}$, and drop the time index from the sensor measurements.

The measurements are sent to the FC whose task is to reconstruct the field of interest. The complete map of the process, obtained from the sensor measurements, is denoted by

$$
\mathbf{U}=\left[u_{i j}\right]_{\substack{i=1, \ldots, I \\ j=1, \ldots, J}}
$$

Most natural phenomena have a compressible (sparse) representation in the frequency domain, and we will assume that this holds for our measurements as well. Specifically, let $\mathbf{V}=\mathbf{W}_{I} \mathbf{U} \mathbf{W}_{J}$ be the two-dimensional spatial discrete

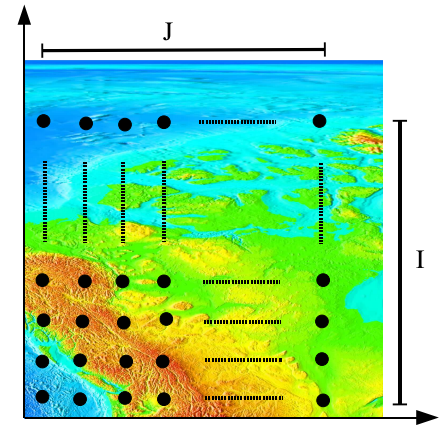

Fig. 1. A grid sensor network consisting of $N=I J$ sensor nodes.

Fourier transform of $\mathbf{U}$, where $\mathbf{W}_{I}$ is the matrix of discrete Fourier transform coefficients, $\mathbf{W}_{I}[m, k]=e^{-j 2 \pi m k / I}$. It can now be shown that $\mathbf{v}=\left(\mathbf{W}_{J} \otimes \mathbf{W}_{I}\right) \mathbf{u}$, where $\mathbf{v}=\operatorname{vec}(\mathbf{V})$ and $\mathbf{u}=\operatorname{vec}(\mathbf{U})$. The Fourier representation $\mathbf{v}$ is assumed to be sparse.

Each sensor node measures the physical quantity of interest and encodes the measurements, along with the sensor's location tag, into a data packet of $L$ bits, which is then modulated and transmitted to the FC. Upon reception, the FC demodulates the signal and extracts the measurement information. The communication noise in the channel may cause one or more bits to be received in error. Without loss of generality, we assume that a packet is in error if one or more bits are determined to be in error, i.e., we assume that no coding is employed. ${ }^{1}$ If a packet is determined to be in error (via errordetection, i.e., CRC), the FC discards that packet. Assuming that the system has bandwidth $B$ and that each sensor transmits at a bit-rate equal to the bandwidth, the packet duration is $T_{p}=\frac{L}{B}$.

The observations of a random subset of $M$ sensors' packets at the FC can be expressed as

$$
\mathbf{y}=\mathbf{R u}+\mathbf{z}
$$

where $\mathbf{R}$ is an $M \times N$ random selection matrix, consisting of $M$ rows of the identity matrix selected uniformly at random, and $\mathbf{z}$ represents the sensing noise. ${ }^{2}$ Noting that $\mathbf{u}=\Psi_{\mathbf{v}}$, where $\boldsymbol{\Psi}=\left(\mathbf{W}_{J} \otimes \mathbf{W}_{I}\right)^{-1}$ is the inverse Discrete Fourier Transform matrix, Eq. (2) can be re-written in terms of the

\footnotetext{
${ }^{1}$ Coding will improve the packet error rate and can easily be incorporated into our analysis once a code is specified

${ }^{2}$ Note the distinction between the sensing noise, $\mathbf{z}$, which arises due to the limitations in the sensing device, and the communication noise, which is a characteristic of the transmission system. The sensing noise $\mathbf{z}$ appears as an additive term in Eq. (2), whereas the communication noise results in bit errors and packet loss. The communication noise thus affects the structure of the random selection matrix $\mathbf{R}$ but does not appear as an additive term in the observation vector $\mathbf{y}$.
} 
sparse vector $\mathbf{v}$ as

$$
\mathbf{y}=\mathbf{R} \Psi_{\mathbf{v}}+\mathbf{z}
$$

To reconstruct the field, the FC first tries to recover the vector $\mathbf{v}$ as accurately as possible, then uses it to construct the map $\mathbf{U}$. Given the observations $\mathbf{y}$, the random selection pattern $\mathbf{R}$ and the sparsity basis $\Psi$, and in the absence of sensing noise $\mathbf{z}$ - which is the case we will be focusing on - reconstruction can be performed by solving the following minimization problem:

$$
\operatorname{minimize}_{\tilde{\mathbf{v}}}\|\tilde{\mathbf{v}}\|_{\ell_{1}} \quad \text { subject to } \mathbf{R} \Psi \tilde{\mathbf{v}}=\mathbf{y} .
$$

The theory of compressed sensing (specifically, [15]) states that as long as the number of observations, picked uniformly at random, is greater than $N_{s}=C S \log N$, then with very high probability the solution to the convex optimization problem (4) is unique and is equal to $\mathbf{v}$. Here $C$ is a constant that is independent of $N$ and $S$ (see [15] for the details). Thus, in our case, it suffices to ensure that the FC collects at least $N_{s}$ packets picked uniformly at random from different sensors to guarantee accurate reconstruction of the field with very high probability.

\section{PACKet ARrival Process}

The nodes in a RACS scheme transmit asynchronously, whenever they have a packet. As in any random access, packets may collide at the FC. The key idea behind RACS is to let the FC simply discard the colliding packets and those packets that were received in error due to communication noise. This approach is motivated by the compressed sensing theory, i.e., the fact that the FC does not care which specific sensors are selected as long as (i) the selected subset is chosen uniformly at random, and (ii) there are sufficiently many collision-free and error-free packets received to allow for the reconstruction of the field. Therefore, so long as the packet collisions and packet errors occur randomly, discarding of those packets will not change the way in which the FC perceives the arrival of useful packets. Note that the matrix $\mathbf{R}$ of Eq. (3) includes the effects of packet loss due to both collisions and errors, i.e., $M$ represents the number of useful packets received by the FC after the erroneous and colliding packets are discarded.

Assuming no channel coding, a packet will be in error if one or more of its bits are in error. The probability of packet error is thus given by

$$
P_{E}=1-\left(1-P_{e}\right)^{L}
$$

where $P_{e}$ is the probability of bit error and $L$ is the number of bits per packet. The probability of bit error is related to the signal-to-noise ratio (SNR) $\gamma$, e.g., $P_{e}=\frac{1}{2} e^{-\gamma}$ for DPSK [16].

In what follows, we consider a Poisson process for packet generation at each node, and analyze the aggregate arrival process of useful packets at the FC. We then study the conditions under which this process yields a sufficient number of measurements, $N_{s}=C S \log N$. These conditions in turn imply a per-node packet generation rate, $\lambda_{1}$, which fully defines the continuous-time RACS.

\section{A. Useful Packets}

We assume that each node generates packets according to an independent Poisson process at an average rate of $\lambda_{1}$ packets per second. The overall packet generation rate is $\lambda=N \lambda_{1}$. In order to reconstruct the field, the FC needs to collect at least $N_{s}$ useful packets; however, there is no guarantee that all the arriving packets will be useful. Three factors contribute to packet discarding: collisions, errors, and repetitions. The latter refers to a situation in which the $\mathrm{FC}$ receives more than one packet from the same node during a collection interval $T$. In such a case, the FC keeps only one packet and discards the copies (the copies carry the same information since the measurement field is assumed not to have changed during one collection interval).

The FC buffers the useful packets, and waits to have sufficiently many to perform the reconstruction. The total number of packets that are used in the reconstruction process, $K\left(\lambda_{1}, T\right)$, is thus the number of received packets left after discarding the colliding packets, the erroneous packets, and multiple copies of the same packet. We conjecture that the arrival of useful packets is a Poisson process with an effective average arrival rate $\lambda^{\prime} \leq \lambda$, given by

$$
\lambda^{\prime}=\frac{N\left(1-e^{-\lambda_{1} T}\right) e^{-2 N \lambda_{1} T_{p}}\left(1-P_{E}\right)}{T}
$$

The effective arrival rate given by Eq. (6) can be justified by modeling the collisions, the packet errors and the repetitions as follows.

(i) Given an aggregate generation rate $\lambda=N \lambda_{1}$, the probability of no collision at the $\mathrm{FC}$ is modeled as

$$
\operatorname{Prob}\{\text { no collision }\}=e^{-2 N \lambda_{1} T_{p}}
$$

The probability of no packet error is given by

$$
\operatorname{Prob}\{\text { no packet error }\}=1-P_{E}
$$

(ii) The probability of no repetition is assessed by focusing on an individual node. Let $\mathcal{N}_{1}(T)$ denote the number of packets that a given node generates in $T$. If this number is greater than 1, the FC will discard the repetitions. Hence, the number of "non-repeated" packets generated at each node during $T$ is given by

$$
\mathcal{N}_{1}^{\prime}(T)= \begin{cases}0, & \mathcal{N}_{1}(T)=0 \\ 1, & \mathcal{N}_{1}(T) \geq 1\end{cases}
$$

The average effective packet generation rate at each node is thus some $\lambda_{1}^{\prime} \leq \lambda_{1}$. Let us define the corresponding reduction factor as $\rho=\lambda_{1}^{\prime} / \lambda_{1}$, i.e.,

$$
\begin{aligned}
\rho & =\frac{E\left\{\mathcal{N}_{1}^{\prime}(T)\right\}}{E\left\{\mathcal{N}_{1}(T)\right\}}=\frac{1-\operatorname{Prob}\left\{\mathcal{N}_{1}(T)=0\right\}}{\lambda_{1} T} \\
& =\frac{1-e^{-\lambda_{1} T}}{\lambda_{1} T}
\end{aligned}
$$

The average arrival rate of useful packets at the $\mathrm{FC}, \lambda^{\prime}$, can now be expressed as the product of the average aggregate arrival rate $N \lambda_{1}$, the probability of no collision, the probability 
of no packet error, and the reduction factor $\rho$ :

$$
\lambda^{\prime}=N \lambda_{1} e^{-2 N \lambda_{1} T_{p}}\left(1-P_{E}\right) \rho
$$

Substituting for $\rho$ from Eq. (7), the above expression reduces to (6). The Poisson model for $K\left(\lambda_{1}, T\right)$ is now given by

$$
\operatorname{Prob}\left\{K\left(\lambda_{1}, T\right)=k\right\}=P_{K}\left(k ; \lambda_{1}, T\right)=\frac{\left(\lambda^{\prime} T\right)^{k}}{k !} e^{-\lambda^{\prime} T}
$$

In order to examine our conjecture, we conducted numerical experiments. Fig. 2 shows the histogram of a simulated $K\left(\lambda_{1}, T\right)$ process. Shown also in the figure are the Poisson distribution functions with conjectured $\lambda^{\prime}$ of Eq. (6), and with $\lambda_{\text {est }}$, an average arrival rate estimated from the simulated data. Noting a good match between the conjectured model and the simulation, we adopt the model (9) and proceed to design the system in Section IV.

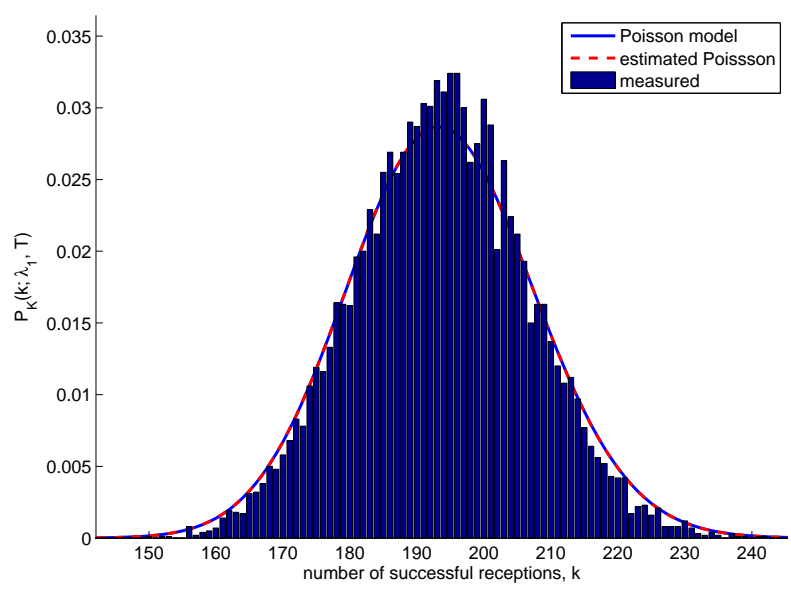

Fig. 2. Probability distribution function of the number of useful packets $K\left(\lambda_{1}, T\right)$ for $N=2500, T=1000 \mathrm{~s}, \lambda_{1}=10^{-4}$ packet $/ \mathrm{s}, T_{p}=0.2 \mathrm{~s}$ and $P_{E}=0.1$; the number of simulation runs is 10,000 .

\section{SYSTEM DESIGN}

The parameters that define a RACS system with $N$ nodes are the per-node packet generation rate $\lambda_{1}$, the collection interval $T$, the bit rate $B$ which determines the packet duration $T_{p}=L / B,{ }^{3}$ and the probability of packet error $P_{E}$. These parameters define $\lambda^{\prime}$ through Eq. (6). System design involves finding a set of parameters for which a performance requirement is met. Specifically, we define a performance requirement based on the concept of sufficient sensing.

\section{A. Probability of Sufficient Sensing}

In order to perform field reconstruction, the FC needs to collect $N_{s}=C S \log N$ or more useful packets during the time interval $T$. However, because the packet arrival process is random, there is no guarantee that the $\mathrm{FC}$ will collect sufficiently many packets during this interval. We thus define

\footnotetext{
${ }^{3}$ Without loss of generality, throughout this paper we use bandwidth and bit rate interchangeably
}

the probability of sufficient sensing as the probability that the FC collects $N_{s}$ or more useful packets during $T$, and we specify the performance requirement as the minimum probability of sufficient sensing, $P_{s}$. In other words, we ask that the FC collect at least $N_{s}$ useful packets during time $T$ with probability $P_{s}$ or higher. This condition can be expressed as

$$
\operatorname{Prob}\left\{K\left(\lambda_{1}, T\right) \geq N_{s}\right\} \geq P_{s}
$$

Given the Poisson model for the number of useful packets $K\left(\lambda_{1}, T\right)$, we have that

$\operatorname{Prob}\left\{K\left(\lambda_{1}, T\right) \geq k\right\}=Q_{K}\left(k ; \lambda_{1}, T\right)=1-\sum_{i=0}^{k-1} P_{K}\left(i ; \lambda_{1}, T\right)$

where $P_{K}\left(k ; \lambda_{1}, T\right)$ is given by Eq. (9). The sufficient sensing requirement (10) is now expressed as

$$
Q_{K}\left(N_{s} ; \lambda_{1}, T\right) \geq P_{s}
$$

where $P_{s}$ is the desired probability of sufficient sensing (which is a system design parameter).

Fig. 3 shows $Q_{K}\left(N_{s} ; \lambda_{1}, T\right)$ versus $\alpha=\lambda^{\prime} T$ for two different values of $N_{s}$. These values correspond to $N=2500$ nodes, and $S=10$ or 16 , with $C=2$. For a given $N_{s}$ and a desired probability of sufficient sensing $P_{s}$, one can find the corresponding $\alpha_{s}$ as illustrated in the figure. The soobtained $\alpha_{s}$ represents the number of useful packets that have to be acquired during the collection interval $T$ in order to meet the sufficient sensing probability $P_{s}$. In other words, the condition (12) can equivalently be stated as

$$
\alpha \geq \alpha_{s}
$$

where $\alpha_{s}$ is a design target that depends on $N_{s}$ and $P_{s}$ only, and can be determined through the procedure illustrated in Fig. 3.

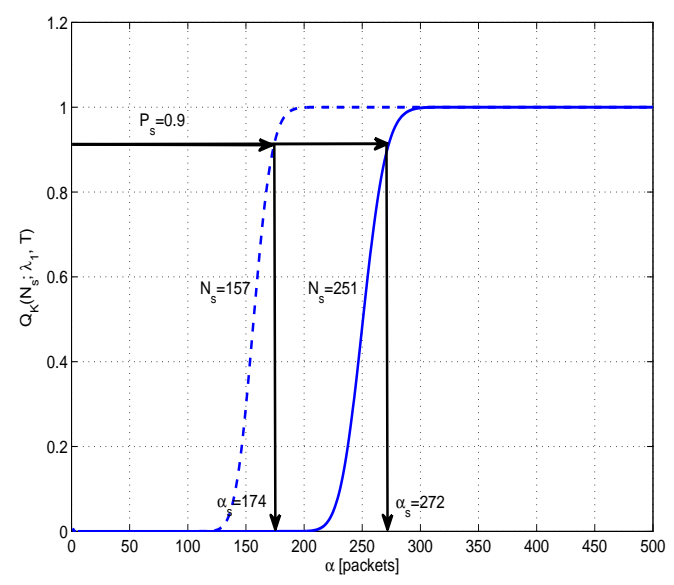

Fig. 3. $Q_{K}\left(N_{s} ; \lambda_{1}, T\right)$ vs. $\alpha=\lambda^{\prime} T$ for $N_{s}=157$ and 251 packets. A required probability of sufficient sensing $P_{s}$ implies a corresponding minimum value of $\alpha, \alpha_{s}$. 


\section{B. Design objective}

The design objective is to determine the per-node packet generation rate $\lambda_{1}$ that is necessary to ensure sufficient sensing. In doing so, we will assume that $T$ and $L$ are set apriori, as these parameters pertain to the actual process being sensed ( $T$ is determined in accordance with the desired update rate and has to be less than $T_{c o h}$, while $L$ is determined by the nature of the measurements). We will also assume that the probability of packet error $P_{E}$ is fixed, i.e., the system is designed to provide a certain SNR $\gamma_{0}$ at the receiver, or equivalently, a certain probability of bit error $P_{e}$.

Fig. 4 shows $\alpha$ versus $\lambda_{1}$ for a given set of system parameters $N, P_{S}, T, L$ and $\gamma_{0}$. We note from the figure that depending upon the bandwidth $B$, a solution for $\lambda_{1}$ may or may not exist. If sufficient bandwidth is available such that a solution exists, then the range of acceptable values for $\lambda_{1}$ is the set of values for which $\alpha \geq \alpha_{s}$. Within this range, we choose the smallest value of $\lambda_{1}$, as it corresponds to least frequent transmissions, i.e., it minimizes the overall energy consumption as we will discuss in Section V.

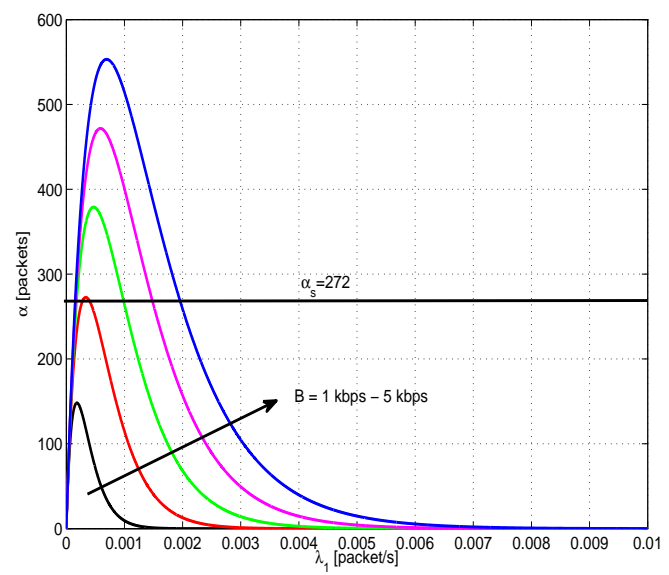

Fig. 4. Average number $\alpha$ of useful packets received during collection time $T$ is plotted vs. $\lambda_{1}$ using Eq. (6) for different values of $B$ and for $\alpha_{s}=272$ $\left(N_{s}=251, P_{s}=0.9\right)$. Other system parameters are $T=1000 \mathrm{~s}, L=1000$ bits and $P_{E}=0.1\left(\gamma_{0}=9.2 \mathrm{~dB}\right.$ assuming uncoded DPSK $)$. This figure shows that in order to achieve a desired $\alpha_{s}$ a certain minimum bandwidth is required. When sufficient bandwidth is available, the desired value of $\alpha_{s}$ implies the minimum required per-node packet rate $\lambda_{1 s}(B)$.

\section{Design Example}

We use a numerical example to demonstrate the design procedure and to visually illustrate the field recovery process using RACS. We consider a $50 \times 50$ grid network measuring a physical process with a spatial map given in Figure 6(a). This map may for instance represent the temperature. Its spatial Fourier transform indicates a sparse behavior with sparsity $S=16$. Let us assume a collection time $T=1000 \mathrm{~s}$, a packet size $L=1000$ bits, a bit rate $B=5 \mathrm{kbps}$ and a packet error probability $P_{E}=0.1$. From Fig. 3 the value of $\alpha_{s}$ is determined as $\alpha_{s}=272$ packets. Fig. 5 shows a zoomed-in version of $\alpha$ versus $\lambda_{1}$ according to Eq. (6). Using this figure, the desired value of $\alpha_{s}$ obtained from Fig. 3 determines the sufficient per node sensing rate to be $\lambda_{1 s}=1.52 \times 10^{-4}$ packet/s for $B=5 \mathrm{kbps}$. Employing the so-obtained sensing rate, Figure 6(b) shows the image recovered by the FC after $T=1000 \mathrm{~s}$. We note an excellent similarity between the original and the recovered map. The normalized error for the recovered image $\left(\frac{\|\hat{\mathbf{u}}-\mathbf{u}\| \ell_{\ell_{2}}}{\|\mathbf{u}\|_{\ell_{2}}}\right)$ is on the order of $10^{-10}$ which is in the domain of numerical round-off errors.

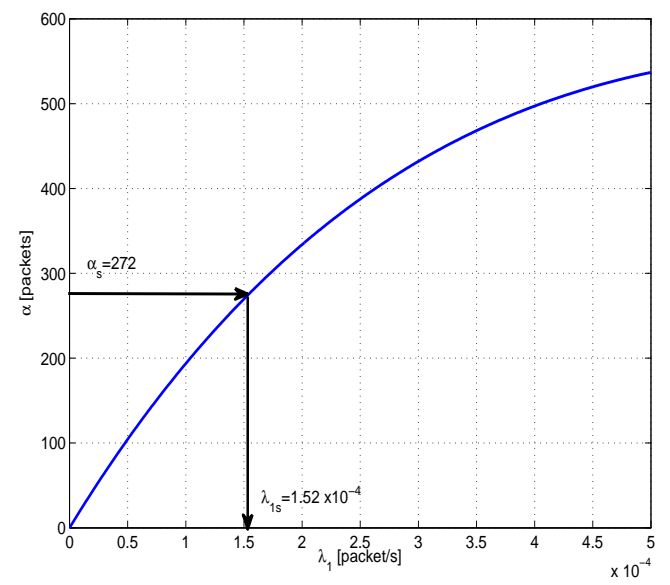

Fig. 5. The value of $\alpha_{s}=272$ obtained from Fig. 3 determines a per node sufficient sensing rate $\lambda_{1 s}=1.52 \times 10^{-4}$ packet/s.

\section{BANDWIDTH AND ENERGY CONSIDERATIONS}

\section{A. Minimum Bandwidth}

The observations that we made from Fig. 4 imply that in order for a set of design parameters to satisfy the sufficient sensing condition, a minimum bandwidth is required. For example, we see in Fig. 4 that given $\alpha_{s}=272$ packets, for $B=1 \mathrm{kbps}$ no value of $\lambda_{1}$ can meet the sufficient sensing requirement (13), while for $B=2 \mathrm{kbps}$ a solution to $\lambda_{1}$ exists. The minimum required bandwidth is obtained by identifying the maximum of $\alpha$, i.e., by taking the derivative of $\alpha$ with respect to $\lambda_{1}$ and setting it to zero. The value of $\lambda_{1}$ at which the maximum occurs is given by

$$
\lambda_{1 x}=\frac{1}{T} \log \left(1+\frac{T}{2 N L / B}\right)
$$

and the corresponding maximum value of $\alpha$ is given by

$$
\alpha_{\max }=\frac{B T}{2 L}\left(1+\frac{B T}{2 N L}\right)^{-2 N L / B T-1}\left(1-P_{E}\right)
$$

In order for a solution to exist, this value has to be

$$
\alpha_{\max } \geq \alpha_{s}
$$

which implies that the system bandwidth $B$ has to be greater than some minimum, $B_{s}$. Eq. (16) can be solved numerically to obtain the minimum required bandwidth $B_{s}$. While there is no closed form solution for $B_{s}$, an approximation can be 


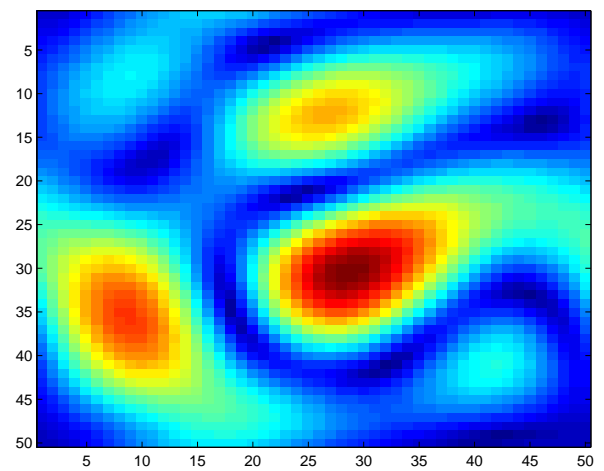

(a) The original map of the sensing field.

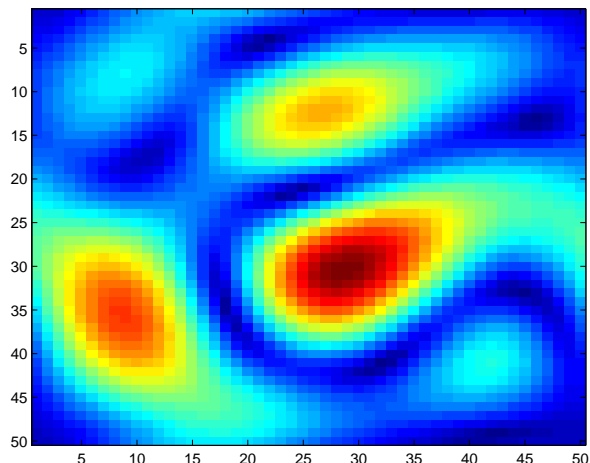

(b) The recovered map of the sensing field.

Fig. 6. The sensing field is recovered employing RACS with $\lambda_{1 s}=1.52 \times$ $10^{-4}$ packet/s, for $T=1000 \mathrm{~s}, P_{E}=0.1$ and $T_{p}=0.2 \mathrm{~s}$.

obtained when $2 N L / T B \gg 1$ :

$$
B_{s} \approx \frac{2 e L \alpha_{s}}{T\left(1-P_{E}\right)}
$$

Fig. 7 shows the exact and the approximate values of $B_{s}$ versus $N$ for a given set of parameters. The exact values for $B_{s}$ are obtained by solving Eq. (16) numerically, whereas the approximate values follow from Eq. (17). We note that the approximation serves as a lower bound on $B_{s}$, and that it becomes quite tight as $N$ grows.

\section{B. Energy Consumption}

In a battery-powered network where re-charging is difficult, as is the case in underwater deployments, network lifetime is of utmost importance. Energy per successfully delivered bit of information thus naturally emerges as a figure of merit for system performance. In light of a sensor network based on compressed sensing, we define a related figure of merit as the total average energy required for one field reconstruction:

$$
E=N \lambda_{1} T \cdot P_{T} \cdot T_{p}
$$

where the first term $\left(N \lambda_{1} T\right)$ is the average number of nodes that transmit in one collection time $T ; P_{T}$ is the per-node

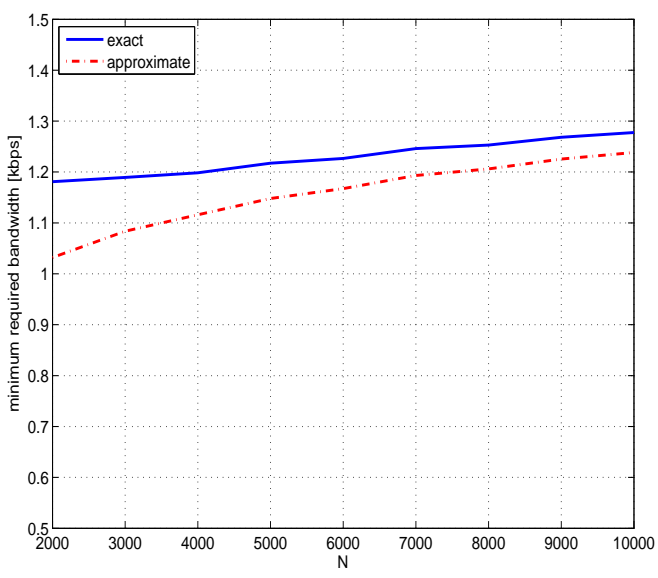

Fig. 7. Minimum required bandwidth $B_{s}$ vs. the number of nodes $N$. The system parameters are $S=10, P_{s}=0.9, L=1000$ bits, $T=1000 \mathrm{~s}$ and $P_{E}=0.1$

transmit power, and $T_{p}$ is the packet duration, i.e., the time during which a node is active. In a system designed to provide a certain SNR $\gamma_{0}$ at the receiver, choosing the bandwidth automatically determines the necessary transmit power. Assuming an AWGN channel with the noise power spectral density $N_{0}$ and attenuation $A$ between the transmitter and the receiver, the SNR is given by

$$
\gamma=\frac{P_{T} / A}{N_{0} B}
$$

Without loss of generality, we assume the same attenuation $A$ for all the links. Hence, the transmit power of each node is $P_{T}=\gamma_{0} A N_{0} B$, and we have that

$$
E=N \lambda_{1} T \gamma_{0} A N_{0} L
$$

As expected, the energy consumption is minimized if one chooses the minimum sensing rate $\lambda_{1 s}(B)$.

Fig. 8 shows $\lambda_{1 s}$ versus $B$ for a given set of system parameters $N, L, T$, and $\gamma_{0}$. We note from this figure that $\lambda_{1 s}(B) \leq \lambda_{1 s}\left(B_{s}\right)$ whenever the solution exists, i.e., for $B \geq B_{s}$. Thus $\lambda_{1 s}\left(B_{s}\right)$ can be used to provide an upper bound on the energy expenditure for a RACS network. Using the expression (14) we have that

$$
\lambda_{1 s}\left(B_{s}\right)=\frac{1}{T} \log \left(1+\frac{T}{2 N L / B_{s}}\right)
$$

Hence, the energy (20) is bounded by

$$
E_{u p}=N \log \left(1+\frac{T}{2 N L / B_{s}}\right) \cdot \gamma_{0} A N_{0} L
$$

Finally, using Eq. (17) a closed form approximation for the upper bound on the energy consumption can be obtained as

$$
E_{u p} \approx N \log \left(1+\frac{e \alpha_{s}}{N\left(1-P_{E}\right)}\right) \cdot \gamma_{0} A N_{0} L
$$

Fig. 9 shows the energy consumption (20) of the RACS 
network (normalized with respect to $A N_{0}$ ) for $B=2 \mathrm{kbps}$ and $B=10 \mathrm{kbps}$, as well as the exact and approximate upper bounds given by Eqs. (22) and (23), respectively. The minimum bandwidth that determines the bound is $B_{s} \approx 1 \mathrm{kbps}$.

Note also that $\lambda_{1 s}$ decreases with bandwidth, reaching a limiting value as $B \rightarrow \infty$ (see Fig. 8). This value can be analytically determined as

$$
\lambda_{1}(\infty)=\frac{1}{T} \log \left(\frac{1}{1-\frac{\alpha_{s}}{N\left(1-P_{E}\right)}}\right)
$$

which can be used to assess a lower bound on the energy consumption,

$$
\begin{aligned}
E_{\text {low }} & =N \lambda_{1}(\infty) T \gamma_{0} A N_{0} L \\
& =N \log \left(\frac{1}{1-\frac{\alpha_{s}}{N\left(1-P_{E}\right)}}\right) \cdot \gamma_{0} A N_{0} L
\end{aligned}
$$

The lower bound (25) is included in Fig.9. We note that this bound is well approached already with $B=10 \mathrm{kbps}$.

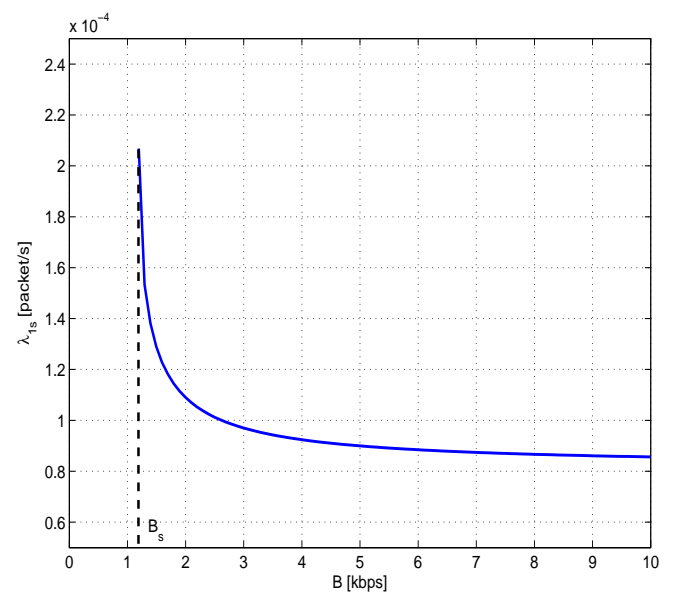

Fig. 8. Minimum per-node packet rate $\lambda_{1 s}$ vs. $B$ for $N=2500, L=1000$ bits, $T=1000 \mathrm{~s}$ and $P_{E}=0.1$. Note that $\lambda_{1 s}(B) \leq \lambda_{1 s}\left(B_{s}\right)$ for $B \geq B_{s}$.

\section{COMPARISON BENCHMARK}

As a comparison benchmark, we assume a conventional network with deterministic sensing and deterministic access. In the conventional network, all $N$ nodes conduct measurements and transmit their data packets to the FC using a standard time-division multiple access (TDMA). This approach requires scheduling, such that packets from different nodes arrive backto-back at the FC. Fig. 10 depicts the required scheduling process.

In the presence of channel noise, packet errors are bound to occur. To deal with the packet errors, the conventional network can either employ automatic repeat request (ARQ), or allow for some packet loss (no ARQ). In what follows we consider both scenarios.

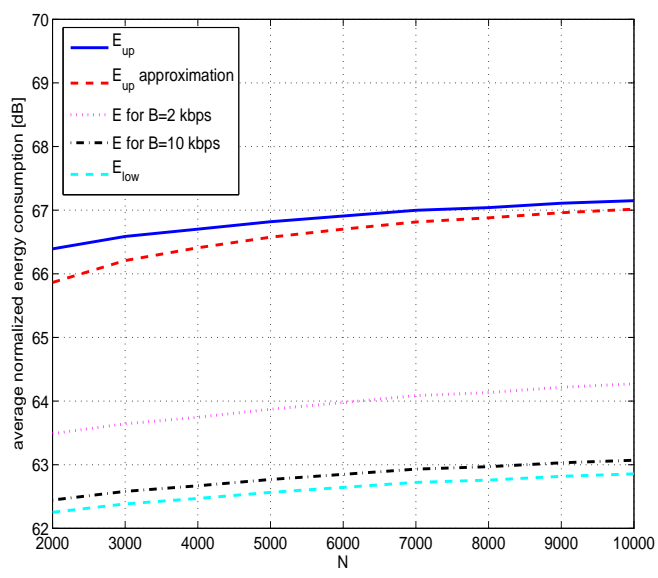

Fig. 9. Average normalized energy consumption vs. the number of nodes $N$. The system parameters are $P_{s}=0.9, S=10, L=1000$ bits, $T=1000 \mathrm{~s}$ and $P_{E}=0.1\left(\gamma_{0}=9.2 \mathrm{~dB}\right.$ assuming uncoded DPSK).

\section{A. $A R Q$}

To achieve reliable data transmission in a conventional network, if a data packet is determined to be in error, the FC sends a re-transmission request to the node whose packet is received in error. The extra time it takes to collect the retransmitted packets extends the overall collection time. The average number of times a packet needs to be re-transmitted before it is successfully received is $1 / 1-P_{E}$. In a full-duplex system it thus takes $T=N T_{p} /\left(1-P_{E}\right)$ on the average to transmit all $N$ packets. ${ }^{4}$ For a specified updating interval $T$, the bandwidth required by the system is

$$
B_{c, A R Q}=\frac{N L}{T\left(1-P_{E}\right)}
$$

The average energy consumption of a conventional network with ARQ is given by

$$
E_{c, A R Q}=P_{T} T=\frac{N}{1-P_{E}} \cdot \gamma_{0} A N_{0} L
$$

\section{B. No $A R Q$}

Unlike the ARQ scheme, this scheme does not compromise the collection time. However, once a packet is detected to be in error, complete reconstruction is not achieved. Hence, the probability of correct frame reception in this scheme parallels the sufficient sensing probability in RACS. The probability that the FC receives all $N$ packets correctly during one collection interval is $\left(1-P_{E}\right)^{N}$. Equating this probability with an equivalent sufficient sensing requirement implies that

$$
\left(1-P_{E}\right)^{N} \geq P_{s}
$$

In other words, we ask that full reconstruction occur with probability of at least $P_{s}$. Alternatively, we may relax this

\footnotetext{
${ }^{4}$ In a half-duplex underwater acoustic network, this time must also include the waiting time which depends on the propagation delay. The propagation delay in turn depends on the distance between the FC and the sensor nodes and can be rather long due to the low speed of sound.
} 


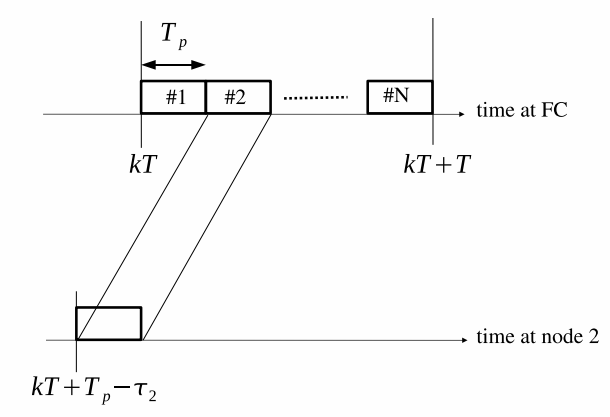

Fig. 10. The scheduling required at each node in the benchmark case of TDMA.

requirement and ask instead that a fraction of packets, say $N_{x}=X \% N$, be received correctly. Then, we want

$$
\sum_{n=N_{x}}^{N}\left(\begin{array}{l}
N \\
n
\end{array}\right)\left(1-P_{E}\right)^{n} P_{E}^{N-n} \geq P_{s}
$$

Given a design target $P_{s}$, the condition (28) implies that $P_{e} \leq P_{e}^{*}$, where the threshold $P_{e}^{*}$ can be computed numerically. For a given functional dependence $P_{e}=f(\gamma)$, the corresponding bit SNR condition becomes $\gamma \geq \gamma^{*}=f^{-1}\left(P_{e}^{*}\right)$. For example, if $N_{x}=N$, assuming that

$$
\left(1-P_{E}\right)^{N} \approx\left(1-L P_{e}\right)^{N} \approx 1-N L P_{e}
$$

we obtain

$$
\gamma \geq \gamma^{*}=f^{-1}\left(\frac{1-P_{s}}{N L}\right)
$$

One frame of data contains $N$ packets, and the total collection time has to be $T \geq N T_{p}$. Thus, the bandwidth required in this case is

$$
B_{c, n o A R Q}=\frac{N L}{T}
$$

The total energy consumed during one collection interval is

$$
E_{c, n o A R Q}=N P_{T} T_{p}=N \gamma^{*} A N_{0} L
$$

For example, using the expression (31) and assuming uncoded DPSK, we obtain

$$
E_{c, n o A R Q}=N A N_{0} L \log \frac{N L}{2\left(1-P_{s}\right)}
$$

We will use this value for performance comparisons in the next section.

\section{PERFORMANCE ANALYSIS}

In this section we quantify the savings in bandwidth and energy that can be achieved through RACS as compared to the conventional scheme of Section VI.

Savings in bandwidth can be quantified as

$$
B_{c, A R Q} / B_{s} \approx \frac{N}{2 e \alpha_{s}}=G_{B 1}
$$

and

$$
B_{c, n o A R Q} / B_{s} \approx \frac{N\left(1-P_{E}\right)}{2 e \alpha_{s}}=G_{B 2}
$$

where we have used the approximation for the minimum bandwidth of a RACS network given by Eq. (17).

Savings in energy can be quantified as

$$
E_{c, A R Q} / E_{u p} \approx \frac{1}{\left(1-P_{E}\right) \log \left(1+\frac{e \alpha_{s}}{N\left(1-P_{E}\right)}\right)}=G_{E 1}
$$

and

$$
E_{c, n o A R Q} / E_{u p} \approx \frac{\log \frac{N L}{2\left(1-P_{s}\right)}}{\gamma_{0} \log \left(1+\frac{e \alpha_{s}}{N\left(1-P_{E}\right)}\right)}=G_{E 2}
$$

where we have used the upper bound on energy consumption for RACS given by Eq. (23).

Now, to quantify a lower bound on the energy savings obtained by RACS, let us assume that the packet errors caused by noise do not affect the performance of the benchmark case, i.e., the conventional network can perfectly recover the field regardless of the packet errors. The conventional network thus has a minimum energy consumption given by

$$
E_{c}=N P_{T} T_{p}=N \gamma_{0} A N_{0} L
$$

This provides us with the minimum savings that RACS can achieve:

$$
E_{c} / E_{u p} \approx \frac{1}{\log \left(1+\frac{e \alpha_{s}}{N\left(1-P_{E}\right)}\right)}=G_{E \min }
$$

Fig. 11 shows the saving in bandwidth plotted versus the size of the network. This figure demonstrates that RACS is capable of providing a factor of two in bandwidth savings for our example network with $N=2500$ nodes, or more for a larger network (assuming that the sparsity level $S$ remains the same). This is a significant feature from the viewpoint of a band-limited system.

Fig. 12 shows the corresponding energy saving versus the size of the network. We note that RACS offers substantial savings over the benchmark case. For example, for $N=2500$ nodes, the energy consumed by RACS is about $9 \mathrm{~dB}$ less than the energy consumed by the benchmark TDMA network of the same size, not employing ARQ. If ARQ is employed in the benchmark network, the savings are almost $7 \mathrm{~dB}$. Even if we assume the benchmark case does not suffer from any packet errors, RACS can still offer more than $6 \mathrm{~dB}$ savings in energy.

\section{CONCLUSION}

We proposed a networking scheme that combines the concepts of random channel access and compressed sensing to achieve energy and bandwidth efficiency, and to eliminate any synchronization or scheduling requirements. The scheme is suitable for large coverage networks, deployed for longterm monitoring of phenomena that is sparse in the spatialfrequency domain. Network design principles were provided 


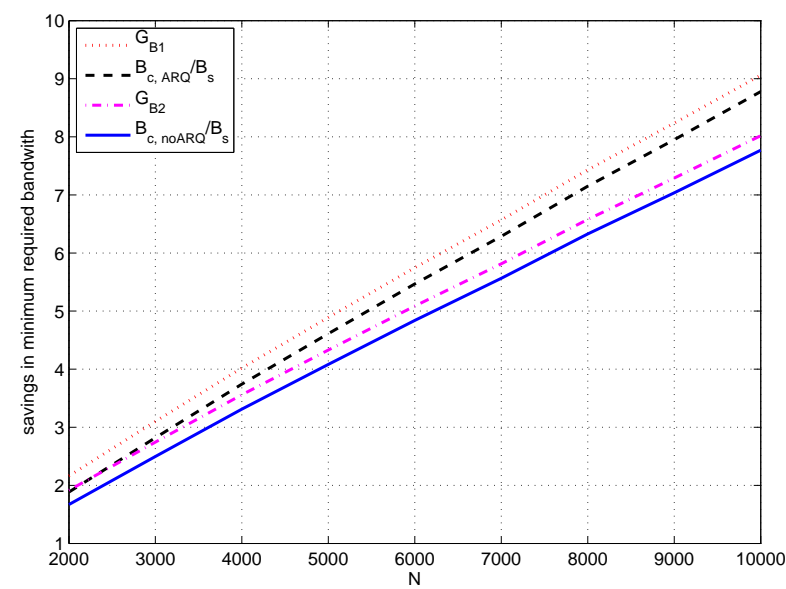

Fig. 11. The saving in minimum required bandwidth vs. the number of nodes $N$, for $P_{E}=0.1$. The rest of the system parameters are $S=10, P_{s}=0.9$, $L=1000$ bits, $T=1000 \mathrm{~s}$.

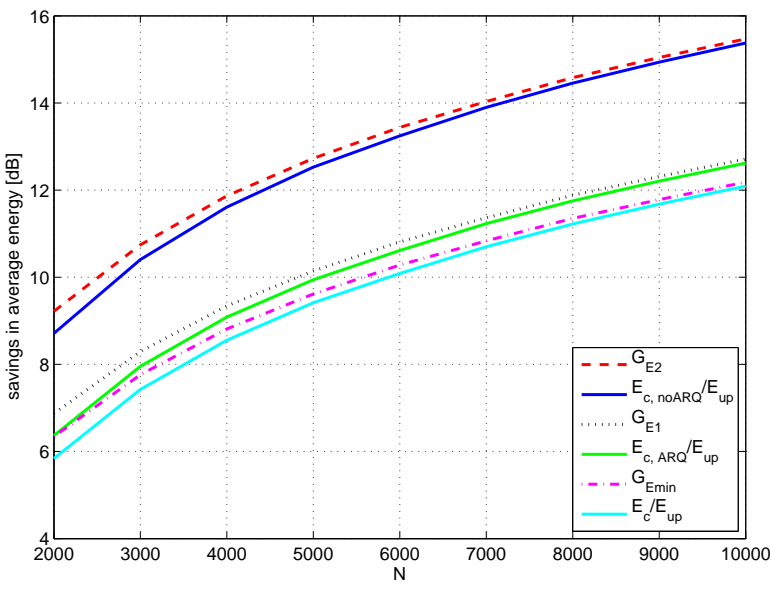

Fig. 12. Total saving in the average energy vs. the number of nodes $N$ for $P_{E}=0.1$. The rest of the system parameters are $S=10, P_{s}=0.9$, $L=1000$ bits, $T=1000 \mathrm{~s}$.

based on the concept of sufficient sensing probability. With this probability, which is the system design target, the fusion center is guaranteed to acquire sufficiently many observations to reconstruct the measured field during a given interval of time. A desired probability of sufficient sensing in turn implies the necessary rate at which each sensor node should transmit its measurements. (A probabilistic approach is necessary in order to account for packet loss due to both collisions and bit errors caused by communication noise.) The performance of RACS was assessed analytically in terms of the energy and bandwidth requirements, demonstrating substantial savings over a conventional scheme based on deterministic channel access and sensing. Future work will focus on inclusion of channel fading effects.

\section{REFERENCES}

[1] K. Sohrabi, J. Gao, V. Ailawadhi, and G. J. Pottie, "Protocols for selforganization of a wireless sensor network," IEEE Personal Communications, vol. 7, pp. 16-27, October 2000.

[2] C. Schurgers, V. Tsiatsis, S. Ganeriwal, and M. Srivastava, "Optimizing sensor networks in the energy-latency-density design space," IEEE Transactions on Mobile Computing, vol. 1, no. 1, pp. 70-80, 2002.

[3] W. Wei, J. Heidemann, and D. Estrin, "An energy-efficient mac protocol for wireless sensor networks," in IEEE Infocom, June 2002, pp. 1567 1576.

[4] M. Zorzi, P. Casari, N. Baldo, and A. Harris III, "Energy-efficient routing schemes for underwater acoustic networks," IEEE Journal on Selected Areas in Communications, vol. 26, no. 9, pp. 1754-1766, December 2008.

[5] F. Zorzi, M. Stojanovic, and M. Zorzi, "On the effects of node density and duty cycle on energy efficiency in underwater networks," in IEEE Oceans'10 Asia Pacific Conference, May 2010.

[6] E. J. Candes and M. B. Wakin, "An introduction to compressive sampling," IEEE Signal Processing Magazine, pp. 21-30, March 2008.

[7] R. Baraniuk, "Compressive sensing," IEEE Signal Processing Magazine, pp. 118-121, July 2007.

[8] W. Bajwa, J. Haupt, A. Sayeed, and R. Nowak, "Compressive wireless sensing," in 5th Int. Conf. Information Processing in Sensor Networks (IPSN'06), April 2006, pp. 134-142.

[9] W. Bajwa, A. Sayeed, and R. Nowak, "Matched source-channel communication for field estimation in wireless sensor networks," in 4th Int. Conf. Information Processing in Sensor Networks (IPSN'05), April 2005, pp. 332-339.

[10] W. Bajwa, J. Haupt, A. Sayeed, and R. Nowak, "Joint source-channel communication for distributed estimation in sensor networks," IEEE Transactions on Information Theory, vol. 53, no. 10, pp. 3629-3653, October 2007.

[11] Q. Ling and Z. Tian, "Decentralized sparse signal recovery for compressive sleeping wireless sensor networks," IEEE Transactions on Signal Processing, vol. 58, no. 7, pp. 3816-3827, July 2010.

[12] Y. Mostofi and P. Sen, "Compressive cooperative sensing and mapping in mobile networks," in Proceedings of the American Control Conference (ACC), St. Louis, Missouri, June 2009.

[13] S. Poduri, G. Marcotte, and G. S. Sukhatme, "Compressive sensing based lightweight sampling for large robot groups." [Online]. Available: http://cres.usc.edu/pubdb_html/files_upload/647.pdf

[14] F. Fazel, M. Fazel, and M. Stojanovic, "Random access compressed sensing in underwater acoustic networks," in Forty-Eight Annual Allerton Conference on Communication, Control and Computing, September 2010.

[15] E. J. Candes, J. Romberg, and T. Tao, "Robust uncertainty principles: Exact signal reconstruction from highly incomplete frequency information," IEEE Transactions on Information Theory, vol. 52, pp. 489-509, March 2006.

[16] J. G. Proakis, Digital Communications, 3rd ed. McGraw Hill, 1995. 